\title{
Localization of Noncovalent Complexes in MALDI-Preparations by CLSM
}

\author{
Verena Horneffer, ${ }^{*}$ Kerstin Strupat, and Franz Hillenkamp \\ Institute for Medical Physics and Biophysics, University of Münster, Münster, Germany
}

\begin{abstract}
The unambiguous detection of noncovalent complexes (NCCs) by matrix-assisted laser desorption/ ionization mass spectrometry (MALDI-MS) is still a far cry from being routine. For protein NCCs such as their quaternary structure it has been reported that signals of the intact complex are only obtained for the first or at most the first few laser exposures of a given sample area. This observation was called the first-shot phenomenon. In the present study, this first-shot phenomenon has been investigated for the hexameric protein complex allophycocyanine (APC) by two independent methods, MALDI-MS with a (nearly) pH-neutral matrix 6-aza-2-thiothymine (6-ATT) and by imaging the fluorescence of the complex in APC-6-ATT preparations by confocal laser scan microscopy (CLSM). The intact APC heterohexamer loses its visible fluorescence upon dissociation into its subunits. Both methods consistently show that intact APC complexes are precipitated at the matrix crystal surface, but dissociate upon incorporation into the matrix crystals. (J Am Soc Mass Spectrom 2006, 17, 1599-1604) (c) 2006 American Society for Mass Spectrometry
\end{abstract}

$\mathrm{M}$ any biological processes are governed by the specific interactions of biopolymers with each other. The investigation of the formation of noncovalent complexes is, thus, of general interest in modern science. Despite the wide spread applications of MALDI-MS for proteomics, noncovalent complexes (NCCs) of proteins and other molecules of biological significance are mainly analyzed by electrospray ionization (ESI-MS) [1-4]. From early on, it appeared that the MALDI process does not lend itself easily to the unambiguous detection of many, if not most, noncovalent complexes. Nevertheless, the detection of NCCs by MALDI-MS has been reported in several publications [5-11]. A physiological $\mathrm{pH}$ of the matrix is generally considered conducive to the detection of specific complex signals, but signals of complexes have also been obtained with acidic matrices such as ferulic or nicotinic acid.

Several attempts have been made to develop an unambiguous protocol for the detection of intact NCCs of large proteins by MALDI-MS. The problem is complicated by the fact that in MALDI spectra, one frequently observes signals of nonspecific complexes, formed most probably in the plume upon laser desorption [12]. As one of the unambiguous criteria, it was postulated that the signal of the NCC must be more intense than that of any of its components. Replacement of one of the components of the complex by a less specific one has also been used to verify the specificity of the recorded signal $[6,8]$. Rosinke et al. observed a so-called first-shot phenomenon [13], which

Published online August 14, 2006

Address reprint requests to Dr. V. Horneffer, Institute of Biomedical Optics, University of Lübeck, Peter-Monnik-Weg 4, D-23562 Lübeck, Germany. E-mail: horneffer@bmo.uni-luebeck.de

* Current address: Institute of Biomedical Optics, University of Lübeck, Peter-Monnik-Weg 4, D-23562 Lübeck, Germany. has been verified in several other publications [14-16]. The first-shot phenomenon describes the observation that signals of specific noncovalent protein complexes are often detected as base peak in MALDI-mass spectra for the first or the first few exposures of a given sample spot only, whereas the signals of the single subunits dominate the spectra of subsequent irradiations [14]. There are several different possibilities to explain this observation. One holds that the NCCs are incorporated homogeneously into the crystal lattice but the penetrating UV radiation of the first laser shot destroys the NCCs in deeper layers. This possibility was excluded by Rosinke et al. [13], who exposed the samples to a high dose of CW UV radiation without affecting the intact NCCs seen in the first-shot spectra. Another explanation assumes that the NCCs dissociate upon inclusion into the matrix crystals leaving only intact complexes precipitated at the crystal surface. This explanation was suggested by Cohen et al. [14]. These authors assume that intact NCCs on the crystal surface still have sufficient interaction with the matrix to be successfully desorbed and ionized. Zehl et al. challenged this concept with the suggestion that the laser pulse energy is the most crucial parameter for the successful detection of large protein NCCs [17, 18]. They suggested that NCCs are incorporated into the bulk crystals, but NCCs on surfaces are not completely surrounded by matrix. Thus, less energy is necessary to desorb (and ionize) complexes from the surface as compared to those fully incorporated [18]. An increased laser pulse energy leads to a harsher desorption process, which is much more unfavorable for the detection of intact NCCs. It should be noted that the first-shot phenomenon is not observed for all analyte-matrix combinations and not for complexes such as protein-DNA interaction or DNA-DNA interaction, e.g., double helices, [11, 19-22]. 
In a separate publication, it was shown that confocal laser scanning microscopy (CLSM) can be used to localize proteins in matrix crystals, if covalently labeled with a suitable, $\mathrm{pH}$-independent fluorophore, e.g., Texas Red [23]. As the fluorophore is covalently labeled to a single analyte molecule, this labeling technique, unfortunately, does not discriminate between specific and nonspecific analyte interactions. To overcome these difficulties, allophycocyanine (APC), a noncovalent $107 \mathrm{kDa}$ protein heterohexamer, was chosen as analyte molecule in the present study for MALDI-MS and independent CLSMimaging because it exhibits specific fluorescence properties in dependence of its quaternary structure [24-27] and shows the first-shot phenomenon when analyzed in a 6-aza-2-thiothymine matrix.

In this study, MALDI-MS and CLSM are used to investigate the localization of intact APC as an example for a large protein noncovalent complex. The analyte distribution found gives evidence for the explanation of the first-shot phenomenon of large protein NCCs attributable to analyte precipitation at the crystal surfaces as introduced by Cohen et al.

\section{Materials and Methods}

\section{Materials}

The matrices 6-azo-2-thiothymine (6-ATT) and 2,5-dihydroxybenzoic acid (2,5-DHB) were obtained from SigmaAldrich, Steinheim, Germany. 2,5-DHB was purified following the protocol described before [23]. Both matrices were prepared as aqueous solutions at a concentration of $10 \mathrm{~g} / 1$ in doubly distilled water $\left(\mathrm{H}_{2} \mathrm{O}_{\text {bidest }}\right)$. 2,5-DHB was used in a mixture of 9 parts 2,5-DHB and 1 part 2-hydroxy-5-methoxybenzoic acid (Sigma-Aldrich) at $10 \mathrm{~g} / 1$ solved in pure ethanol (Merck, Darmstadt, Germany) (DHBs). Texas Red (TR) labeled avidin (TR-avidin) was supplied by Molecular Probes (Invitrogen, Breda, The Netherlands). A mass spectrometric analysis ascertained that TR was covalently bound to single avidin subunits and that no free dye molecules were present in the samples. The sample was dissolved in $\mathrm{H}_{2} \mathrm{O}_{\text {bidest }}$ at a concentration of $10^{-4} \mathrm{M}$. Allophycocyanin (APC) was obtained from ProZyme (San Leonardo, CA) in a buffered solution with a concentration of $1.6^{*} 10^{-4} \mathrm{M}$. The buffer system of the original sample solution was exchanged against ammonium acetate buffer with a concentration of $25 \mathrm{mM}$ and pH 7.5, which is more convenient for further MALDI-analysis. The resulting APC-solution was stored at $4{ }^{\circ} \mathrm{C}$ till further use. BODIPY495/503 from Molecular Probes was used to stain the standard immersion oil to enhance the contrast of the CLSM-images.

\section{Preparations}

Single crystals of 6-ATT were grown by evaporation of solvent from a saturated solution of 6-ATT in $\mathrm{H}_{2} \mathrm{O}_{\text {bidest }}$. Seven hundred fifty $\mu \mathrm{l}$ of this solution was mixed with an appropriate amount of the analyte solution to achieve the desired molar analyte-to-matrix ratio (A/M-ratio). The final solution was stored open in a dark, quiet location for several days until crystals had grown. The crystals were separated from the mother liquor by filtration, washed with $4{ }^{\circ} \mathrm{C}$ cold $\mathrm{H}_{2} \mathrm{O}_{\text {bidest, }}$ dried, and immediately imaged by CLSM. For this purpose, the single crystals were placed on a microscope slide. A cavity around the crystals was formed by double-sided adhesive tape. This cavity was filled with BODIPY495/503 dyed standard immersion oil and closed with a cover slip. Dried-droplet preparations were prepared by mixing adequate volumes of matrix and analyte solutions to achieve the desired A/M-ratio either on a metal target for MALDI-MS, or on a cover slide in case CLSM-imaging was performed in addition to the MALDI analysis.

\section{Instrumentation}

For the mass spectrometry a modified VISION 2000 (Finnigan MAT, Bremen, Germany) was used in the reflectron mode with $12 \mathrm{kV}$ acceleration voltage in the ion source and $20 \mathrm{kV}$ post acceleration for ion detection. The mass spectrometer was equipped with a nitrogen laser (LSI/Spectra Physics/Newport, Mountain View, CA) with a wavelength of $337 \mathrm{~nm}$. Laser pulse energy was adjusted slightly above ion detection threshold to achieve a better signal-to-noise ratio in a single mass spectrum. For further improvement of this ratio, up to 20 single mass spectra of the given setting but from different spots were accumulated. A Leica TCS (Wetzlar, Germany) was used for the confocal laser scanning microscopy (CLSM) to image the sample fluorescence. The instrument was equipped with an Ar-Kr-laser, and a 63x-oil-immersion objective with a numerical aperture of 1.32. For TR-avidin measurements, the excitation wavelength was set to 568 $\mathrm{nm}$ while fluorescence above a wavelength of $590 \mathrm{~nm}$ was detected. $647 \mathrm{~nm}$ was used as excitation wavelength for APC, while the emission was detected above a wavelength of $665 \mathrm{~nm}$. A CLSM shadowgraph was obtained by exciting the BODIPY495/503 fluorescence at a wavelength of $488 \mathrm{~nm}$ and imaging its fluorescence in the wavelength range between $515 \mathrm{~nm}$ and $545 \mathrm{~nm}$. The excitation irradiance was adjusted to avoid photobleaching during data acquisition. The size of the pinhole was set to a diameter of $75 \%$ of the Airy disk in the image plane and a 4- to 8 -fold frame averaging was used to optimize data acquisition. A RF-5001PC spectrofluorometer (Shimadzu, Europe, Duisburg, Germany) was used to measure the fluorescence of APC in different solutions.

\section{Results and Discussion}

The detection of the intact NCC of APC by MALDI-MS with a 6-ATT matrix is demonstrated in Figure 1a. It shows the mass spectrum of a dried-droplet preparation with only first shots onto a given spot summed. In this mass spectrum, the hexamer is the base peak of the spectrum, while the distribution of singly charged subunits and multiply charged complexes shows the MALDI- 
(a)

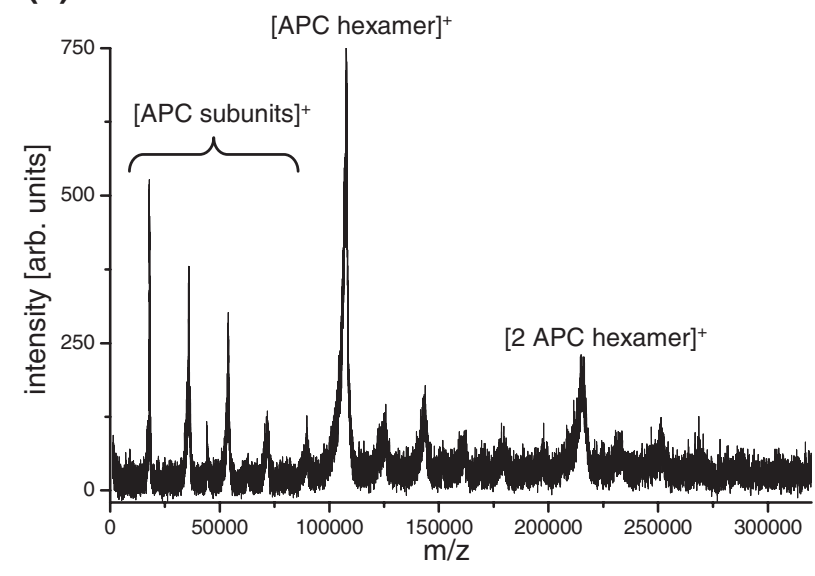

(b)

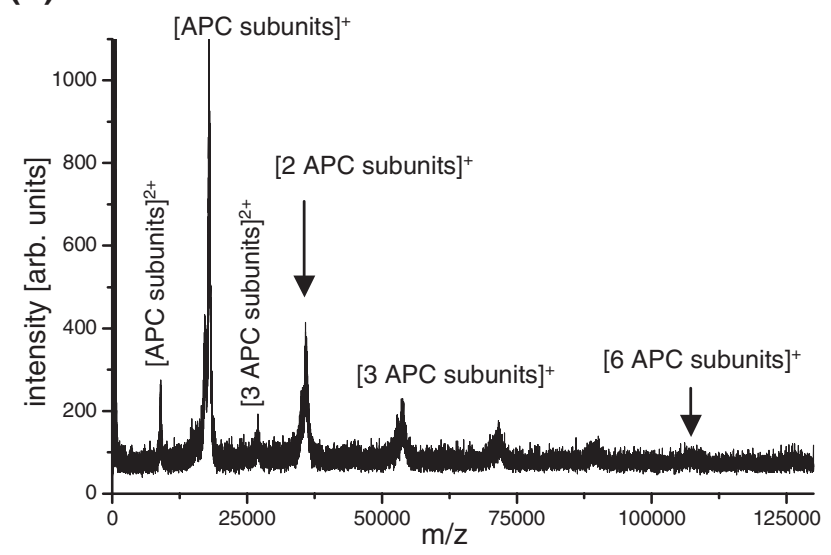

Figure 1. Mass spectra of 6-ATT dried-droplet preparation with APC as analyte molecules. (a) Only first shots onto 20 different spots were accumulated. The APC hexamer is the base peak in the mass spectrum. (b) Second and up to 30 following shots onto a given spot were accumulated. Peaks of single APC subunits and their nonspecific multimers are the most intense peaks in the mass spectrum.

specific decrease in intensity of unspecific analyte aggregations. In this spectrum, even the signal of the dodecamer, which represents the unspecific dimer of the (native) hexamer, is detected at an intensity well above that of the rest of the (unspecific) oligomers. For the mass spectrum of Figure $1 \mathrm{~b}$ the second and up to 30 following shots onto a given spot were accumulated. The peaks of the single subunits are the most intense peaks in this mass spectrum and the intensity of the (mostly nonspecific) hexamer fits the expected exponential decline of signal intensity with increasing mass. The outstanding intensity of the hexamer in the mass spectrum of Figure 1a, taken only from first shots onto a given spot, is interpreted as evidence of a specific noncovalent complex and representing the first-shot phenomenon [13-15]. Only subunits of APC could be observed using DHBs as matrix (data not shown).

In a former study, it had been shown that certain matrix substances, e.g., 2,6-dihydroxybenzoic acid, ex- clude all analyte molecules from their crystals lattice [23, 28]. In a first step the incorporation behavior of 6-ATT was, therefore, investigated using the same protocol as given by Horneffer et al. [23]. Single crystals of 6-ATT were grown by evaporation of the solvent. Texas Red (TR) labeled avidin was added to the solution to result in a molar A/M-ratio of $2.2^{*} 10^{-5}$. Figure 2 shows representative confocal xy- and xz-sections of a 6-ATT-single crystal doped with TR-avidin. The images on the left side show the fluorescence of the immersion oil stained with BODIPY495/503 to outline the crystal shapes. For the interpretation of Figure 2c, it must be kept in mind that the crystal casts a shadow in the z-direction attributable to optical effects such as refraction and birefringence. Therefore, the bottom edge is difficult to identify and is indicated in the figure by a dashed line. The right-hand sided images show the fluorescence of TR-avidin. The line in Figure $2 \mathrm{~b}$ indicates the location of the $\mathrm{xz}$-scan, seen in Figure $2 \mathrm{~d}$ and vice versa. Comparing the images, two important details can be observed. On the one hand, TR-fluorescence can be detected clearly out of the inner bulk of the crystal. This indicates that 6-ATT incorporates proteins into its crystal lattice. On the other hand, an increased fluorescence intensity can observed at the crystal edges. Unfortunately, within this experiment it cannot be differentiated whether this increase derives from optical effects as observed earlier [23] or from augmented protein attachment at the matrix crystal surfaces.

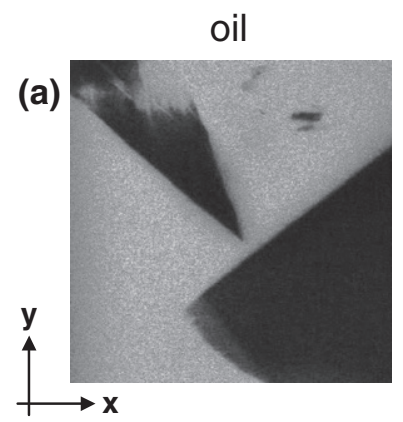

(b)
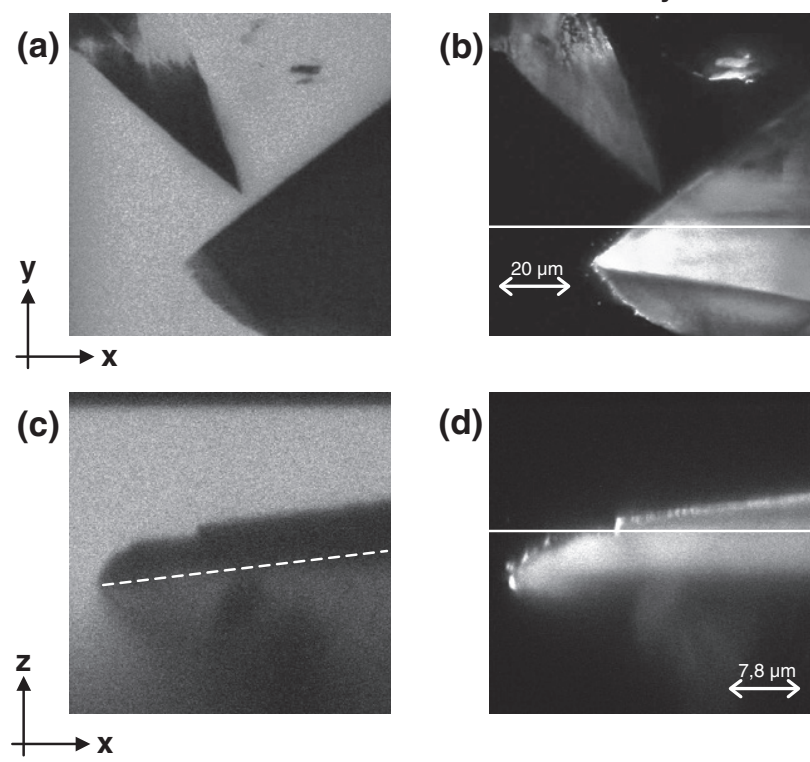

(d)

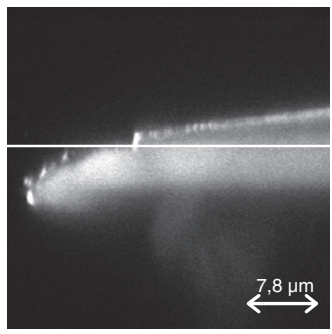

Figure 2. CLSM-xy- and xz-sections of 6-ATT-single crystals doped with TR-avidin. The molar A/M-ratio was $2,2^{*} 10^{-5}$; (a) and (b) show xy-sections, (c) and (d) xz-sections. The images on the left show the BODIPY495/503-immersion oil fluorescence, the images on the right the fluorescence of TR-avidin. The line in (b) represents the location of the xz-scan presented in (d), and in (d) the location of the xy-scan, respectively. The dashed line in (c) indicates the bottom edge of the crystal as it is difficult to identify. See scale bars for dimension. 

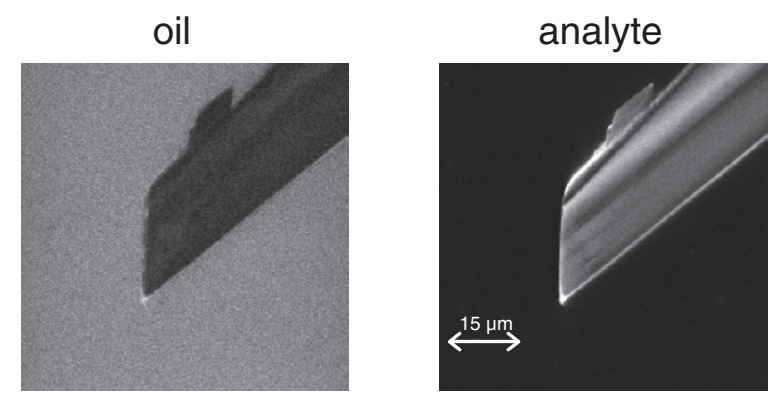

Figure 3. CLSM-xy-section of a dried-droplet preparation of 6-ATT with TR-avidin. The molar A/M-ratio was $3^{*} 10^{-5}$. The left image on the left shows the BODIPY495/503-immersion oil fluorescence, image on the right the fluorescence of TR covalently bound to avidin subunits. See scale bar for dimension.

To ascertain that analyte incorporation takes place not only for slowly growing single crystals but also for standard dried-droplet preparations crystals from the latter were CLSM imaged as well. Figure 3 shows a confocal xy-section through a representative 6-ATT-crystal of a dried-droplet preparation with TR-avidin as analyte molecule and a molar A/M-ratio of $3^{*} 10^{-5}$. Again, the left image shows the shape of the crystal and the right image the fluorescence of TR. The observations are similar to those of single crystals, i.e., analyte signals from the bulk volume of the crystals are recorded, which indicates the incorporation of analyte molecules into the crystal lattice. The congruent incorporation behavior of single crystals and crystals grown from dried-droplet preparation is in good agreement to earlier investigations [23]. A more detailed discussion of the inhomogeneous fluorescence of doubly refracting crystals in an immersion liquid, the enhanced fluorescence from edges in particular, is given in that reference. The main results of this experiment can be summarized as: (1) fluorescence signals from the bulk volume of 6-ATT-crystals prove that 6-ATT incorporates protein analyte molecules, and (2) fluorescence intensity appears increased at the crystal edges. Unfortunately, the fluorescence of TR-avidin does not permit a differentiation between the specific formation its NCCs, in this case the homotetramer TR-avidin, and the dissociation into the subunits or their unspecific agglomeration. For this purpose, the specific fluorescence properties of APC offer an interesting alternative.

APC was chosen as a NCC for this study because of its specific fluorescence properties, which can serve as an independent control of the quaternary structure [24-27]. The NCC of APC is a homotrimer of three heterodimers resulting in an hexamer. The heterodimer consists of two subunits, an $\alpha$-chain of $17,260 \mathrm{Da}$ and a $\beta$-chain of 17,344 Da. Each of the subunits contains a phycocyanobilin molecule bound covalently as prosthetic group. APC exhibits different fluorescence properties, depending on the conformation and because of different interactions of the phycocyanobilin tetrapyrrole chromophores with each other. In the hexamer the prosthetics groups of two neighboring heterodimers are in close enough proximity to facilitated exciton energy-transfer after fluorescence excitation. This close localization results in an intense maximum of absorption at $650 \mathrm{~nm}$ and a strong fluorescence around $660 \mathrm{~nm}$. This configuration also protects the two prosthetic groups against direct effects of the surrounding medium because they are located inside the NCC $[26,27]$. In acidic solution the hexamer dissociates into the three dimers and on into the single $\alpha$ - and $\beta$-subunits. For the heterodimer, the absorption shifts to $620 \mathrm{~nm}$ and the fluorescence emission to $630 \mathrm{~nm}$. However, in the absence of high concentration of chaotropic salts such as $1 \mathrm{M} \mathrm{NaClO}_{4}$, their dissociation is about two orders of magnitude faster than that of the hexamer, so the heterodimer equilibrium concentration remains negligibly small compared to that of the subunits [29]. Single subunits show a weak absorption in the range of $530 \mathrm{~nm}$ to $650 \mathrm{~nm}$ and negligible fluorescence. Thus, any fluorescence signal excited at $647 \mathrm{~nm}$ and detected above a wavelength of $665 \mathrm{~nm}$ must derive from an intact APChexamer.

To examine the stability of APC, its fluorescence was first measured in different solutions: in $25 \mathrm{mM}$ ammonium acetate buffer ( $\mathrm{pH} 7.5)$, in doubly distilled water $(\mathrm{pH}$ 7.0), in an aqueous 6-ATT-solution (10 g/l, $\mathrm{pH} 5.5)$, and in an aqueous 2,5-DHB-solution ( $10 \mathrm{~g} / \mathrm{l}, \mathrm{pH} 2.4)$. The concentration of the complex was adjusted to $6^{*} 10^{-8} \mathrm{M}$, far above the dissociation constant of approx. $5^{*} 10^{-10} \mathrm{M}[24$, 29]. The fluorescence was excited at a wavelength of 647 $\mathrm{nm}$ and the emission detected in the range above $665 \mathrm{~nm}$, identical to the parameter setting in the CLSM measurements. The results are listed in Table 1 . They show a decreasing fluorescence intensity with decreasing $\mathrm{pH}$, as expected. At the $\mathrm{pH}$ of 5.5 of the 6-ATT solution, the fluorescence indicates that a sizable fraction of the protein is still in the hexameric state whereas no fluorescence can be detected at the $\mathrm{pH}$ of 2.4 of the 2,5-DHB solution at which protein noncovalent complexes are fully dissociated. The fluorescence signal of the APC-hexamer can, therefore, be taken as a measure of the dissociation state in the CLSM images. The pH-dependence as such does not explain the occurrence of the first-shot-phenomenon, in particular as this is observed at the near neutral $\mathrm{pH}$ of matrices such as 6-ATT or the hydroxyacetophenones.

To localize intact NCCs of APC in MALDI samples, CLSM measurements were performed as described before [23]. In a first set of experiments, single crystals of 6-ATT were grown in the presence of APC at a molar A/M-ratio of approximately $10^{-5}$. After harvesting and washing the crystals, they were analyzed by CLSM. Figure 4 shows xyand $x z$-sections of a single such crystal. The left row of

Table 1. Fluorescence of APC in different solutions given in arbitrary units using $647 \mathrm{~nm}$ wavelength for excitation and detecting wavelengths above $665 \mathrm{~nm}$ for emission

\begin{tabular}{lcccc}
\hline & $\begin{array}{c}\text { Buffer, } \\
\mathrm{pH} \mathrm{7.5}\end{array}$ & $\begin{array}{c}\mathrm{H}_{2} \mathrm{O}_{\text {bidest' }} \\
\mathrm{pH} \mathrm{7.0}\end{array}$ & $\begin{array}{c}\text { 6-ATT- } \\
\text { solution, } \\
\mathrm{pH} \mathrm{5.5}\end{array}$ & $\begin{array}{c}\text { 2,5-DHB- } \\
\text { solution, } \\
\mathrm{pH} \mathrm{2.4}\end{array}$ \\
\hline \hline $\begin{array}{l}\text { Fluorescence } \\
\text { [a.u.] }\end{array}$ & 50 & 20 & 5 & 0 \\
\hline
\end{tabular}


oil
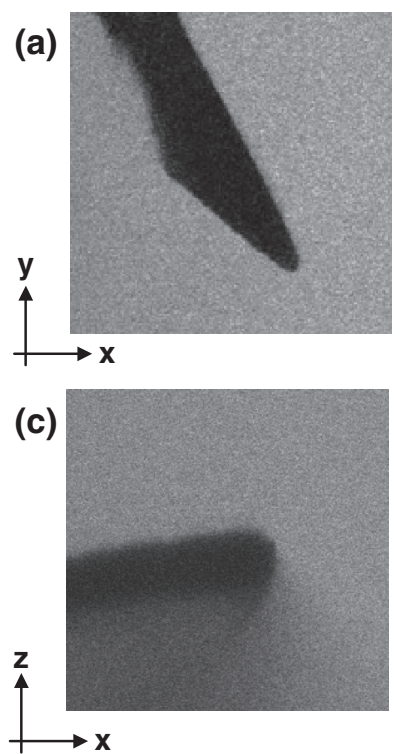

analyte

(b)

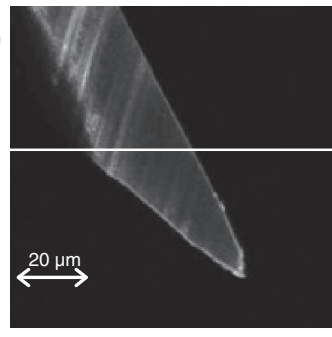

(d)

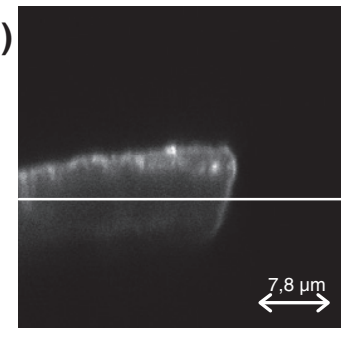

Figure 4. CLSM-xy- and xz-sections of 6-ATT-single crystals doped with APC. The molar A/M-ratio was approx. $10^{-5}$; (a) and (b) show xy-sections, (c) and (d) xz-sections. The images on the left show the BODIPY495/503-immersion oil fluorescence, images on the right the fluorescence of the intact NCC of APC. The line in (b) represents the location of the $x z$-scan shown in the bottom part, and in (d) the location of the xy-scan, respectively. See scale bars for dimension.

images shows the shape of the crystal through the fluorescence of the BODIPY495/503 stained immersion oil. The right row of images shows the fluorescence the intact NCC of APC. The line in Figure $4 \mathrm{~b}$ indicates the location of the xz-scan (Figure 4d) and vice versa. Comparing oil and analyte images, it is obvious that a fluorescence signal of the intact APC can be detected from the crystal edges only and not from the bulk volume of the crystals. As the crystals in Figure 2 (TR-avidin as analyte molecules) and Figure 4 (APC as analyte molecules) are observed under similar conditions, the lack of fluorescence from the inner part of the crystal in Figure 4 cannot be explained simply by the optical artifacts discussed before [23]. Therefore, this observation is interpreted as a strong indication that intact APC hexamers can only be found at the crystal surface. Unfortunately, no mass spectrometry could be performed on these small and fragile single crystals. Dried-droplet preparations of 6-ATT and APC as analyte were, therefore, investigated in addition.

Figure 5 shows a confocal xy-section of a representative 6-ATT-crystal of a dried-droplet preparation with APC as analyte molecule in a molar A/M-ratio of approximately $10^{-5}$. The left image again shows the shadowgraph of the crystal and the right image the APC fluorescence. The observations are similar to those obtained from single crystals; the fluorescence of intact APC can be identified at the crystal surface only. Because the crystals of this drieddroplet preparation had not been washed, the fluorescence is more intense than that of washed single crystals. The spectra shown in Figure 1 were obtained from such

oil

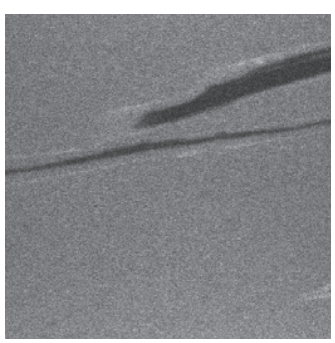

Figure 5. CLSM-xy-sections of dried-droplet preparation of 6-ATT with APC as analyte molecule. The molar A/M-ratio is approximately $10^{-5}$. The image on the left shows the BODIPY495/ 503-immersion oil fluorescence, the image on the right the fluorescence of the intact NCC of APC. See scale bar for dimension.

dried-droplet preparations. Only a very weak, almost negligible signal of the fluorescence of the hexameric APC could be detected at the outer edge of a crystal of an APC doped 2,5-DHB preparation as shown in Figure 6. Mass spectra of these preparations contained only signals of the APC-subunits (data not shown), similar to the spectrum of Figure $1 \mathrm{~b}$ and in agreement with the loss of APC-fluorescence in the 2,5-DHB solution (Table 1).

\section{Conclusions}

The two independent methods of MALDI-MS and CLSM consistently show that the hexameric quaternary structure of the APC-NCC is conserved only for molecules localized at the surface of 6-ATT-matrix crystals. Upon incorporation, both the fluorescence is lost as well as the base signal of the complex in the mass spectrum. These observations support the model presented by Cohen et al. as explanation for the first-shot phenomenon [14]. Although this model has been corroborated by CLSM imaging for only the one complex of APC, there is good reason to assume that it holds as well for other large protein NCCs, for oil

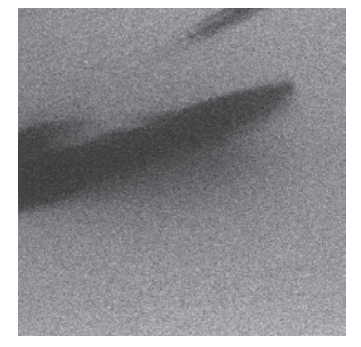

analyte

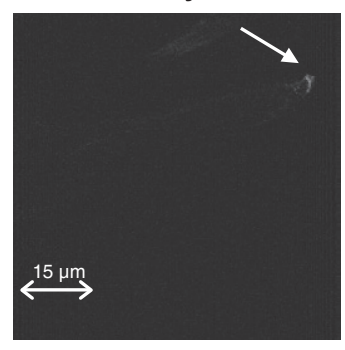

Figure 6. CLSM-xy-sections of dried-droplet preparation of DHBs with APC. The molar A/M-ratio was approximately $10^{-5}$. The image on the left shows the BODIPY495/503-immersion oil fluorescence, the image on the right the fluorescence of the intact NCC of APC. The arrow indicates a location of the small intensity fluorescence of the intact NCC of APC. See scale bar for dimension. 
which the first-shot phenomenon in MALDI-MS is observed.*

At first sight, this result seems to contradict the observation of Zehl et al. who had reported that signals of NCCs of proteins can be obtained for up to 10 exposures of a given sample spot, provided the laser fluence is chosen low enough. This can, however, be rationalized with the notion of a surface localization of the NCCs, if one considers that for fluences very close to ion detection threshold less than a full monolayer is desorbed, particularly if a nonuniform, e.g., near Gaussian, laser beam profile is used. Why NCCs dissociate upon incorporation into matrix crystals is not known, as is the mechanism for incorporation in general. Earlier attempts to find a correlation between the crystal structure and the presence or lack of incorporation have been unsuccessful [28]. The fact that NCCs seem to survive intact only for surface adsorbed species is somewhat surprising, particularly for desorption out of acidic matrices such as nicotinic and ferulic acid [13], because they remain the longest in the low $\mathrm{pH}$ solution. This is also one of the reasons why the desorption of intact protein NCCs by UV-MALDI is so difficult. It has been shown before that analyte molecules, localized strictly at the surface of matrix crystals, experience a significantly harsher desorption as compared to the incorporated ones [30, 31].

The findings reported in this paper cannot be extrapolated to NCCs that contain other than only protein components such as peptides, nucleic acids, etc. For such complexes, the first-shot phenomenon has indeed not been observed. The detection of NCCs by MALDI-MS remains difficult in general. Optimal choice of matrix preparation techniques as well as laser wavelength and fluence must be determined individually for almost each single case. However, in cases where electrospray cannot be used, e.g., because of sample contaminants, MALDI-MS can be used if enough care is taken to optimize the procedure.

\section{Acknowledgments}

The authors gratefully acknowledge Ulrich Kubitscheck, Bonn, Germany, for his technical assistance with the fluorescence microscope and his valuable discussions. This study was done in partial fulfillment of the Ph.D. thesis of $\mathrm{VH}$ in Experimental Physics at the University of Münster.

\section{References}

1. Loo, J. A. Studying noncovalent protein complexes by electrospray ionization mass spectrometry. Mass Spectrom Rev. 1997, 16, 1-23.

2. Loo, J. A. Electrospray ionization mass spectrometry: A technology for studying noncovalent macromolecular complexes. Int. J. Mass Spectrom. 2000, 200, 175-186.

3. Daniel, J. M.; Friess, S. D.; Rajagopalan, S.; Wendt, S.; Zenobi, R. Quantitative determination of noncovalent binding interactions using soft ionization mass spectrometry. Int. J. Mass Spectrom. 2002, 216, 1-27.

\footnotetext{
* At the 54th ASMS conference (2006) in Seattle, WA, USA, Wortmann et al. presented a poster (WP 256) on the detection of protein complexes using the same techniques of MALDI-MS and CLSM imaging as described in this paper. They investigated several protein complexes labeled artifically with FRET fluorochromes. Their findings confirm the results described in this paper.
}

4. Hardouin, J.; Lange, C. M. Biological noncovalent complexes by mass spectrometry. Curr. Organ. Chem. 2005, 9, 317-324.

5. Woods, A. S.; Buchsbaum, J. C.; Worrall, T. A.; Berg, J. M.; Cotter, R. J. Matrix-assisted laser desorption/ionization of noncovalently bound compounds. Anal. Chem. 1995, 67, 4462-4465.

6. Woods, A. S.; Huestis, M. A. A study of peptide-peptide interaction by matrix-assisted laser desorption/ionization. J. Am. Soc. Mass Spectrom. 2001, 12, 88-96.

7. Woods, A. S.; Koomen, J. M.; Ruotolo, B. T.; Gillig, K. J.; Russel, D. H.; Fuhrer, K.; Gonin, M.; Egan, T. F.; Schultz, J. A. A study of peptidepeptide interactions using MALDI ion mobility O-TOF and ESI mass spectrometry. J. Am. Soc. Mass Spectrom. 2002, 13, 166-169.

8. Glocker, M. O.; Bauer, S. H. J.; Kast, J.; Volz, J.; Przybylski, M. Characterization of specific noncovalent protein complexes by UV matrix-assisted laser desorption ionization mass spectrometry. J. Mass Spectrom. 1996, 31, 1221-1227.

9. Kiselar, J. G.; Downard, K. M. Preservation and detection of specific antibody-peptide complexes by matrix-assisted laser desorption ionization mass spectrometry. J. Am. Soc. Mass Spectrom. 2000, 11, 746-750.

10. Zehl, M.; Allmaier, G. Investigation of sample preparation and instrumental parameters in the matrix-assisted laser desorption/ionization time-of-flight mass spectrometry of noncovalent peptide/peptide complexes. Rapid Commun. Mass Spectrom. 2003, 17 1931-1940.

11. Luo, S. H.; Li, Y. M.; Qiang, W.; Zhao, Y. F.; Abe, H.; Nemoto, T.; Qin, X. R.; Nakanishi, H. Detection of specific noncovalent interaction of peptide with DNA by MALDI-TOF. J. Am. Soc. Mass Spectrom. 2004, 15, 28-31.

12. Hillenkamp, F. 1998; Matrix-assisted laser desorption/ionization of noncovalent complexes. Ens, W., Ed.; In New methods for the study of biomolecular complexes; p 181Kluwer Academic Press: The Netherlands,

13. Rosinke, B.; Strupat, K.; Hillenkamp, F.; Rosenbusch, J.; Dencher, N.; Krüger, U.; Galla, H.-J. Matrix-assisted laser desorption/ionization mass spectrometry (MALDI-MS) of membrane proteins and noncovalent complexes. J. Mass Spectrom. 1995, 30, 1462-1468.

14. Cohen, L. R. H.; Strupat, K.; Hillenkamp, F. Analysis of quaternary protein ensembles by matrix assisted laser desorption/ionization mass spectrometry. J. Am. Soc. Mass Spectrom. 1997, 8, 1046-1052.

15. Moniatte, M.; Lesieur, C.; Vécsey-Semjén, B.; Buckley, J. T.; Pattus, F.; van der Groot, F. G.; Van Dorsselaer, A. Matrix-assisted laser desorption-ionization time-of-flight mass spectrometry in the subunit stoichiometry study of highmass noncovalent complexes. Int. I. Mass Spectrom. 1997, 169/170, 179-199.

16. Vogl, T.; Roth, J.; Sorg, C.; Hillenkamp, F.; Strupat, K. Calcium-induced noncovalently linked tetramers of MRP8 and MRP14 detected by ultraviolet matrix-assisted laser desorption/ionization mass spectrometry. J. Am. Soc. Mass Spectrom. 1999, 10, 1124-1130.

17. Zehl, M.; Allmaier, G. Ultraviolet matrix-assisted laser desorption/ionization time-of-flight mass spectrometry of intact hemoglobin complex from whole human blood. Rapid Commun. Mass Spectrom. 2004, 18, 1932-1938.

18. Zehl, M.; Allmaier, G. Instrumental parameters in the MALDI-TOF mass spectrometric analysis of quaternary protein structures. Anal. Chem. 2005, 77, 103-110.

19. Lecchi, P.; Pannell, L. K. The detection of intact double-stranded DNA by MALDI. J. Am. Soc. Mass Spectrom. 1995, 6, 972-975.

20. Gruic-Sovulj, I.; Lüdemann, H. C.; Hillenkamp, F.; Weygand-Duraseciv, I.; Kucan, Z.; Peter-Katalinic, J. Detection of noncovalent tRNA.aminoacyl-tRNA synthetase complexes by matrix-assisted laser desorption/ ionization mass spectrometry. J. Biol. Chem. 1997, 272, 32084-32091.

21. Kirpekar, F.; Berkenkamp, S.; Hillenkamp, F. Detection of doublestranded DNA by IR- and UV-MALDI mass spectrometry. Anal. Chem. 1999, 71, 2334-2339.

22. Distler, A. M.; Allison, J. Additives for the stabilization of doublestranded DNA in UV-MALDI MS. J. Am. Soc. Mass Spectrom. 2002, 13, 1129-1137.

23. Horneffer, V.; Forsmann, A.; Strupat, K.; Hillenkamp, F.; Kubitscheck, $\mathrm{U}$. Localization of analyte molecules in MALDI preparations by confocal laser scanning microscopy. Anal. Chem. 2001, 73, 1016-1022.

24. MacColl, R.; Csatorday, K.; Berns, D. S.; Traeger, E. The relationship of the quaternary structure of allophycocyanin to its spectrum. Arch. Biochem. Biophys. 1981, 208, 42-48.

25. Offner, G. D.; Troxler, R. F. Primary structure of allophycocyanin from the unicellular rhodophyte, Cyanidium caldarium. The complete amino acid sequences of the $\alpha$ and $\beta$ subunits. J. Biol. Chem. 1983, 258, 9931-9940.

26. MacColl, R. Cyanobacterial phycobilisomes. J. Struct. Biol. 1998, 124, 311-334.

27. Ying, L.; Xie, X. S. Fluorescence spectroscopy, exciton dynamics, and photochemistry of single allophycocyanin trimers. J. Phys. Chem. B 1998, 102, 10399-10409.

28. Horneffer, V.; Dreisewerd, K.; Lüdemann, H. C.; Hillenkamp, F.; Lage, M.; Strupat, K. Is the incorporation of analytes into matrix crystals a prerequisite for matrix-assisted laser desorption/ionization mass spectrometry? A study of five positional isomers of dihydroxybenzoic acid. Int. J. Mass Spectrom. 1999, 185/186/187, 859-870.

29. Foguel, D.; Weber, G. Pressure-induced dissociation and denaturation of allophycocyanin at subzero temperatures. J. Biol. Chem. 1995, 270, 28759-28766

30. Glückmann, M.; Pfenninger, A.; Krüger, R.; Thierolf, M.; Karas, M.; Horneffer, V.; Hillenkamp, F.; Strupat, K. Mechanisms in MALDI analysis: Surface interaction or incorporation of analytes? Int. J. Mass Spectrom. 2001, 210, 121-132.

31. Horneffer, V.; Glückmann, M.; Krüger, R.; Karas, M.; Strupat, K.; Hillenkamp, F. Matrix-analyte interaction in MALDI-MS: Pellet and nanoelectrospray preparations. Int. J. Mass Spectrom. 2006, 249/250, 426-432. 